\title{
Postoperative Reduction Quality May Be the Most Important Factor That Causes Worse Functional Outcomes in Open and Closed Pelvic Fractures
}

\author{
Chih-Yang Lai ${ }^{1} \cdot$ Po-Ju Lai ${ }^{1} \cdot$ I-Chuan Tseng $^{2} \cdot$ Chun-Yi Su${ }^{3} \cdot$ Yung-Heng Hsu ${ }^{1}$ • \\ Ying-Chao Chou ${ }^{1} \cdot$ Yi-Hsun $\mathrm{Yu}^{1}$ (i)
}

Accepted: 8 November 2021 / Published online: 1 January 2022

(C) The Author(s) 2021, corrected publication 2022

\begin{abstract}
Background Data on the functional outcomes of patients with open pelvic fractures after osteosynthesis are limited, and whether open fracture is a risk factor for worse outcomes, as compared with closed fracture, remains unclear. This study aimed to compare the functional outcomes of patients with open and closed pelvic fractures and evaluate potential factors that might affect outcomes.

Methods Overall, 19 consecutive patients with open pelvic fractures and 78 patients with closed pelvic fractures between January 2014 and June 2018 were retrospectively reviewed. All fractures were surgically treated, with a minimal follow-up period of three years. Patients' demographic profile, associated injuries, management protocol, quality of reduction, and outcomes were recorded and analyzed.

Results Patients with open pelvic fractures had higher new injury severity score, higher incidence of diverting colostomy, and longer length of stay. Both radiological and functional evaluations revealed no significant differences between the two groups at 1-year and 3-year evaluations. Multiple logistic regression analysis identified poor radiological outcomes (using Lefaivre criteria) and longer length of stay as risk factors for worse short-term functional outcomes. At 3-year evaluation, fair-to-poor radiological outcomes (using Matta/Tornetta and Lefaivre criteria) and the presence of diverting colostomy were potential risk factors.

Conclusions Compared with closed pelvic fracture, open pelvic fracture was not an indicator of worse functional outcomes. Functional outcomes may be comparable between patients with open and closed pelvic fractures at different time points within three years postoperatively. Achieving anatomical reduction in a fracture is crucial, because it might affect patient satisfaction.
\end{abstract}

Yi-Hsun $\mathrm{Yu}$

alanyu1007@gmail.com

1 Department of Orthopedic Surgery, Musculoskeletal Research Center, Chang Gung Memorial Hospital, Linkou Branch, and Chang Gung University, Tao-Yuan, Taiwan. 5, Fu-Hsin St. Kweishan, 33302 Tao-Yuan, Taiwan

2 Department of Orthopedic Surgery, Chang Gung Memorial Hospital, Taoyuan Branch, Tao-Yuan City, Taiwan

3 Department of Orthopedic Surgery, Chang Gung Memorial Hospital, Keelung Branch, Kee-Lung City, Taiwan

\section{Introduction}

Pelvic fractures account for 1\%-3\% of all skeletal injuries, and the management remains challenging for orthopedic surgeons [1]. Significant functional morbidities and a relatively high rate of mortality, ranging from 10 to $16 \%$, may be hypothetically associated with pelvic fractures [2-4]. Therefore, optimal treatment methods to save lives and preserve a satisfactory functional status in patients, following pelvic fracture, are being developed.

Owing to improvements in prehospital care, diagnostic tools, resuscitation protocols, damage control procedures, 
evidence-based algorithms, and multidisciplinary teamwork, the morbidity and mortality rates in patients with pelvic fractures have decreased during the past decade [3, 5-7]. Meanwhile, improvements in the understanding of the pelvic anatomy and concepts of osteosynthesis have led to the expansion of indications for surgical intervention to maximize functional outcomes in these patients [8]. However, several studies have reported that various factors, such as fracture type, sex, age, injury severity score, associated injuries, surgical method, and quality of reduction, might affect post-injury functional outcomes [9-15].

Open pelvic fractures are considered more severe than closed fractures because of the presence of hemodynamic instability, high possibility of soft tissue damage and loss, and high risk of surgical site infection [3, 16, 17]. Functional deficits may theoretically be more significant in patients with open pelvic fractures. To date, only a few studies have addressed the functional outcomes of open pelvic fractures and explored the factors that may cause worse outcomes compared with those of closed fractures. Thus, this study aimed to directly compare functional outcomes after osteosynthesis between open and closed pelvic fractures and investigate the factors that might differentiate outcomes.

\section{Material and methods}

We retrospectively reviewed the medical and radiological records of patients with pelvic fractures who underwent osteosynthesis between January 2014 and June 2018 at our institute from the institutional trauma registry. The review process was approved by the Institutional Review Board (IRB No. 202101095B0).

The inclusion criteria for this study were as follows: (1) patients with pelvic fracture who underwent osteosynthesis, (2) patients who received complete medical and radiological follow-up for at least 12 months, and (3) patients who received complete functional evaluations for more than 36 months. Patients who received conservative treatments, those who had concomitant acetabular fractures, those who were lost to follow-up, and those who did not receive complete functional evaluation were excluded from the study. Patients' demographic profile, injury severity score, new injury severity score, fracture pattern-associated injuries, management protocol, quality of reduction, and outcomes were recorded.

\section{Resuscitation and osteosynthesis protocol}

All patients were resuscitated based on Advanced Trauma Life Support guidelines upon arrival at the emergency department. Since transarterial embolization is a key procedure during resuscitation for pelvic fractures in our institute, it was performed when patients were not responding to blood transfusion and contrast agent pooling, as detected on computed tomography (CT), after the exclusion of other sources of hemorrhage.

Osteosynthesis for pelvic fractures was performed as early as possible when the patient's clinical condition had been stabilized. The surgical approaches and choices of implants for definite treatment were primarily based on the classification of the fracture, distance of fracture displacement, and concomitant visceral or skeletal injuries. Generally, Pfannenstiel and ilioinguinal approaches are the major approaches for ventral ring injury when open reduction and internal fixation (ORIF) are indicated. In cases wherein fracture could be treated via closed reduction and internal fixation (CRIF), percutaneous fixation using screws was performed. External skeletal fixator for ventral ring injury was considered a bridging treatment (between the time of injury and the time of osteosynthesis) or a definite treatment in cases of inaccessible internal fixation from ventral visceral injuries.

For dorsal pelvic ring injury, which is a crescenteric fracture of the iliac wing, sacroiliac joint diastasis, and sacral fracture, CRIF with an iliosacral or trans-iliac-transsacral screw was the first choice for osteosynthesis. However, when anatomical reduction could not be achieved via the closed method or a global instability was present, ORIF using screws or plates or spinopelvic osteosynthesis was indicated and preferred.

\section{Fracture classification and radiological evaluations}

Pelvic fractures were classified, based on the AO/OTA classifications system (2018 revision), to determine the stability of pelvic ring injury, which include the following three types: stable ring, partially stable ring, and unstable ring [18]. Two classification systems were used to identify open pelvic fractures: Faringer and Jones-Powell classifications, which focus on the location of the open wound and the location of the open wound with fracture stability, were specifically used to classify open pelvic fractures [19, 20].

Radiological outcomes were determined through postoperative plain radiographs using three standard pelvic radiographic views: anteroposterior, inlet, and outlet views. Regarding the quality of post-osteosynthesis, we adapted the Matta/Tornetta and the Lefaivre criteria to evaluate vertical displacement and pelvic symmetry, respectively (Table 1) [21-26]. 
Table 1 Grading of radiological outcomes based on Matta/Tornetta and Lefaivre criteria [21]

\begin{tabular}{lll}
\hline & $\begin{array}{l}\text { Matta/Tornetta criteria } \\
\text { Displacement }(\mathrm{mm})\end{array}$ & $\begin{array}{l}\text { Lefaivre criteria } \\
\text { Displacement }(\mathrm{mm})\end{array}$ \\
\hline Excellent & $<4$ & $<5$ \\
Good & $4-10$ & $5-10$ \\
Fair & $10-20$ & $10-20$ \\
Poor & $>20$ & $>20$ \\
\hline
\end{tabular}

\section{Functional evaluations}

Functional outcomes were assessed using Merle d'Aubigné score and Majeed score at 3, 6, 12, 24, and 36 months after injury in all patients. Merle d'Aubigné score includes parameters for pain, mobility, and walking ability, with each parameter rated from 0 points (worst condition) to 6 points (best condition); a high score represents good hip function [27, 28]. Majeed score is a pelvic injury-specific functional assessment that comprises seven items, including pain, work, sitting, sexual intercourse, standing, unaided gait, and walking distance, with a total score range of $0-100$, in order of decreasing disability $[13,29]$. Outcomes were graded as excellent (score $>85$ ), good (score of 70-84), fair (score of 55-69), and poor (score $<55$ ). For statistical analysis, patients were classified into two groups according to the Majeed score: satisfactory outcome, including excellent (score $>85$ ) and good (score of 70-84), and unsatisfactory outcome, including fair (score of 55-69) and poor (score $<55$ ).

\section{Statistical analysis}

Data were analyzed using SPSS software version 23.0 (SPSS Inc., Chicago, IL, USA). Statistical significance was set at a $p$ value of $<0.05$. Continuous variables were compared using Student's $t$ test, whereas categorical variables were compared using the chi-squared test and Fisher's exact test. Multivariate logistic regression was used to

Table 2 Demographic characteristics of 97 patients with pelvic fractures after surgical fixation

\begin{tabular}{|c|c|c|c|}
\hline & Open pelvic fractures & Closed pelvic fractures & $p$ value \\
\hline Number & 19 & 78 & \\
\hline Age (years) & 37.5 (IQR: 28) & 39.6 (IQR: 31 ) & 0.632 \\
\hline Sex, n $(\%)$ & & & 0.308 \\
\hline Men & $10(52.6 \%)$ & $31(39.7 \%)$ & \\
\hline Women & $9(47.4 \%)$ & $47(60.3 \%)$ & \\
\hline \multicolumn{4}{|c|}{ Associate organ injury, n (\%) } \\
\hline Brain & $1(5.3 \%)$ & $12(15.4 \%)$ & 0.453 \\
\hline Chest & $6(31.6 \%)$ & $29(37.2 \%)$ & 0.649 \\
\hline Abdomen & $4(21.1 \%)$ & $18(23.1 \%)$ & 0.850 \\
\hline Urogenital & $5(26.3 \%)$ & $13(16.7 \%)$ & 0.337 \\
\hline Extremities & $12(63.2 \%)$ & $45(57.7 \%)$ & 0.664 \\
\hline ISS (median) & 20 (IQR: 16-27) & 17 (IQR: 8-26) & 0.081 \\
\hline NISS (median) & 27 (IQR: 22-38) & 17 (IQR: 12-30) & 0.010 \\
\hline OTA classification $^{\mathrm{a}}$ & & & 0.058 \\
\hline Stable ring & $4(21.1 \%)$ & $4(5.1 \%)$ & \\
\hline Partially stable ring & $6(31.6 \%)$ & $37(47.4 \%)$ & \\
\hline Unstable ring & $9(47.4 \%)$ & $37(47.4 \%)$ & \\
\hline Osteosynthesis, n (\%) & & & 0.600 \\
\hline ORIF & $13(68.4 \%)$ & $58(74.4 \%)$ & \\
\hline CRIF or ESF & $6(31.6 \%)$ & $20(25.6 \%)$ & \\
\hline Colostomy, n (\%) & $9(47.4 \%)$ & $2(2.6 \%)$ & 0.010 \\
\hline Length of stay (days) & 25.7 (IQR: 11) & 18.5 (IQR: 12.5) & 0.014 \\
\hline Follow up (months) & 39.1 (IQR: 3.5) & 40.6 (IQR: 9) & 0.652 \\
\hline
\end{tabular}

IQR interquartile range, ISS injury severity score, NISS new injury severity score, ORIF open reduction and internal fixation, CRIF closed reduction and internal fixation, ESF external skeletal fixation

${ }^{a}$ The classification of pelvic fracture was based on the AO/OTA classification (2018 revision) 
Table 3 Radiological outcomes and functional outcomes of patients with open and closed pelvic fractures

\begin{tabular}{|c|c|c|c|}
\hline & Open pelvic fractures & Closed pelvic fractures & $p$ value \\
\hline Radiological outcomes & & & 0.425 \\
\hline \multicolumn{4}{|l|}{ Matta/Tornetta criteria } \\
\hline Excellent & $11(57.9 \%)$ & $31(39.7 \%)$ & \\
\hline Good & $5(26.3 \%)$ & $26(33.3 \%)$ & \\
\hline Fair & $3(15.8 \%)$ & $16(20.5 \%)$ & \\
\hline Poor & 0 & $5(6.4 \%)$ & \\
\hline Lefaivre criteria & & & 0.148 \\
\hline Excellent & $8(42.1 \%)$ & $18(23.1 \%)$ & \\
\hline Good & $1(5.3 \%)$ & $20(25.6 \%)$ & \\
\hline Fair & $7(36.8 \%)$ & $24(30.8 \%)$ & \\
\hline Poor & $3(15.8 \%)$ & $16(20.5 \%)$ & \\
\hline \multicolumn{4}{|l|}{ Functional outcomes } \\
\hline \multicolumn{4}{|l|}{ Merle d'Aubigné score } \\
\hline 3 months & 4.7 ((IQR: 5.5) & $5.6((\mathrm{IQR}: 4)$ & 0.295 \\
\hline 6 months & 8.9 (IQR: 6.5) & 9.9 (IQR: 6) & 0.303 \\
\hline 12 months & 12.6 (IQR: 5) & 13.8 (IQR: 4) & 0.192 \\
\hline 24 months & 13.8 (IQR: 4) & 15.2 (IQR: 3) & 0.155 \\
\hline 36 months & 14.4 (IQR: 3.5) & 15.5 (IQR: 4) & 0.231 \\
\hline \multicolumn{4}{|l|}{ Majeed score } \\
\hline 3 months & 32.9 (IQR: 17) & 38.0 (IQR: 23) & 0.179 \\
\hline 6 months & 52.9 (IQR: 30.5) & 55.7 (IQR: 22.5) & 0.538 \\
\hline 12 months & 70.6 (IQR: 19) & 70.9 (IQR: 25) & 0.931 \\
\hline 24 months & 75.0 (IQR: 17) & 78.5 (IQR: 18) & 0.332 \\
\hline 36 months & 76.5 (IQR: 22) & 80.9 (IQR: 20) & 0.239 \\
\hline
\end{tabular}

$I Q R$ interquartile range

determine potential risk factors for unsatisfactory functional outcomes.

\section{Results}

During the 42-month study period, we identified a consecutive series of 97 patients, including 19 patients with open pelvic fractures and 78 patients with closed pelvic fractures who underwent osteosynthesis and met the inclusion criteria. ORIF using a plate was applied in 71 patients, while the other 26 patients underwent CRIF using a screw or an external fixator. For anterior ring injury, the Pfannenstiel approach was applied in 23 patients, and the ilioinguinal approach was applied in 22 patients. Regarding posterior ring injury, the approaches included CRIF with an iliosacral or trans-iliac-trans-sacral screw in 45 patients, ORIF for sacroiliac joint lesions in seven patients, triangular osteosynthesis in two patients, and spinopelvic osteosynthesis in 17 patients.
Patients with open pelvic fractures had a higher new injury severity score ( 27 vs. $17, p=0.01$ ), higher incidence of diverting colostomy (9 [47.4\%] vs. 2 [2.6\%], $p=0.01$ ), and longer hospital stay (25.7 days vs. 18.5 days, $p=0.014$ ) than those with closed fractures (Table 2). However, no significant differences were observed in the number of associated injuries and fracture severity.

Table 3 shows a comparison of radiological and functional outcomes between patients with open pelvic fractures and patients with closed fractures. Although the comparisons of radiological outcomes revealed a higher percentage of malreduction using Matta/Tornetta criteria and equal levels of malreduction using Lefaivre criteria in patients with open pelvic fractures, the comparisons failed to show statistical differences. Similar results were also presented in functional outcome evaluations at each time point.

The associations of the selected factors with the functional outcome (Majeed score) at different time points are demonstrated in Table 4 (1-year evaluation) and Table 5 (3-year evaluation). Moreover, 54 (54.3\%) patients were 
Table 4 Factors associated with short-term (1 year after injury) functional outcomes

\begin{tabular}{|c|c|c|c|}
\hline & $\begin{array}{l}\text { Satisfactory outcome } \\
\text { Majeed score } \geq 70\end{array}$ & $\begin{array}{l}\text { Unsatisfactory outcome } \\
\text { Majeed score }<70\end{array}$ & $p$ value \\
\hline Number & $54(54.3 \%)$ & $43(45.7 \%)$ & \\
\hline Age (years) & 36.5 (IQR: 25) & 42.5 (IQR: 31) & 0.088 \\
\hline \multicolumn{4}{|l|}{ Sex } \\
\hline Men & $20(37.0 \%)$ & $21(48.8 \%)$ & \multirow[t]{2}{*}{0.243} \\
\hline Women & $34(63.0 \%)$ & $22(51.2 \%)$ & \\
\hline Open fracture & $10(18.5 \%)$ & $9(20.9 \%)$ & \multirow[t]{2}{*}{0.766} \\
\hline Closed fracture & $44(81.5 \%)$ & $34(79.1 \%)$ & \\
\hline ISS (median) & 10 (IQR: 7-21) & 24 (IQR: 17-34) & 0.001 \\
\hline NISS (median) & 14 (IQR: 9-25) & 27 (IQR: 18-36) & 0.001 \\
\hline \multicolumn{4}{|l|}{ Associated organ injury } \\
\hline Brain & $3(5.6 \%)$ & $10(23.3 \%)$ & 0.011 \\
\hline Chest & $12(22.2 \%)$ & $23(53.5 \%)$ & 0.001 \\
\hline Abdomen & $10(18.5 \%)$ & $12(27.9 \%)$ & 0.273 \\
\hline Urogenital & $6(11.1 \%)$ & $12(27.9 \%)$ & 0.035 \\
\hline Extremities & $30(55.6 \%)$ & $27(62.8 \%)$ & 0.472 \\
\hline \multicolumn{4}{|l|}{ OTA classification } \\
\hline Stable ring & $7(13.0 \%)$ & $1(2.3 \%)$ & \multirow[t]{3}{*}{0.001} \\
\hline Partially stable ring & $30(55.6 \%)$ & $13(29.4 \%)$ & \\
\hline Unstable ring & $17(31.5 \%)$ & $29(70.6 \%)$ & \\
\hline \multicolumn{4}{|l|}{ Osteosynthesis } \\
\hline ORIF & $38(70.4 \%)$ & $33(76.7 \%)$ & \multirow[t]{2}{*}{0.481} \\
\hline CRIF or ESF & $16(29.6 \%)$ & $10(23.3 \%)$ & \\
\hline \multicolumn{4}{|l|}{ Radiological outcome } \\
\hline \multicolumn{4}{|l|}{ Matta/Tornetta criteria } \\
\hline Excellent & $28(51.9 \%)$ & $14(32.6 \%)$ & \multirow[t]{4}{*}{0.020} \\
\hline Good & $18(33.3 \%)$ & $13(30.2 \%)$ & \\
\hline Fair & $8(14.8 \%)$ & $11(25.6 \%)$ & \\
\hline Poor & 0 & $5(11.6 \%)$ & \\
\hline \multicolumn{4}{|l|}{ Lefaivre criteria } \\
\hline Excellent & $17(31.5 \%)$ & $9(20.9 \%)$ & \multirow[t]{4}{*}{0.048} \\
\hline Good & $15(27.8 \%)$ & $6(14.0 \%)$ & \\
\hline Fair & $16(29.6 \%)$ & $15(34.9 \%)$ & \\
\hline Poor & $6(11.1 \%)$ & $13(30.2 \%)$ & \\
\hline Length of stay (days) & 15.6 (IQR: 9.5) & 25.4 (IQR: 18) & 0.001 \\
\hline Colostomy & $4(7.4 \%)$ & $7(16.3 \%)$ & 0.207 \\
\hline
\end{tabular}

IQR interquartile range, ISS injury severity score, NISS new injury severity score, ORIF open reduction and internal fixation, CRIF closed reduction and internal fixation, $E S F$ external skeletal fixation

satisfied with the 1-year postoperative outcome; however, $43(45.7 \%)$ patients were not (Table 4$)$. The analysis revealed that dissatisfaction was not significantly correlated with the nature of pelvic fracture (open or closed). However, factors, such as injury severity score, new injury severity score, OTA classification, brain injury, chest injury, urogenital injury, application of arterial embolization, length of stay, and radiological outcome, according to the Matta/Tornetta and Lefaivre criteria, seemed to affect 1-year functional performance. Further, multiple logistic regression analysis identified that potential factors, which were related to dissatisfaction, were poor reduction quality (Lefaivre criteria) (odds ratio [OR] 3.55, 95\% confidence interval [CI] 1.07-11.76) and length of stay (OR 1.09, 95\% CI 1.04-1.16) (Table 6).

Similar functional outcomes were noted at the 3-year evaluation, as shown in Table 5. In addition, 80 (82.5\%) patients were satisfied with the outcome; however, 17 
Table 5 Results of factors associated with mid-term (3 years after injury) functional outcomes

\begin{tabular}{|c|c|c|c|}
\hline & $\begin{array}{l}\text { Satisfactory outcome } \\
\text { Majeed score } \geq 70\end{array}$ & $\begin{array}{l}\text { Unsatisfactory outcome } \\
\text { Majeed score }<70\end{array}$ & $p$ value \\
\hline Number & $80(82.5 \%)$ & $17(17.5 \%)$ & \\
\hline Age (years) & 38.3 (IQR: 30) & 43.5 (IQR: 28) & 0.261 \\
\hline Sex men & $36(45.0 \%)$ & $5(29.4 \%)$ & 0.237 \\
\hline Women & $44(55.0 \%)$ & $12(70.6 \%)$ & \\
\hline Open fracture & $14(17.5 \%)$ & $5(29.4 \%)$ & 0.314 \\
\hline Closed fracture & $66(82.5 \%)$ & $12(70.6 \%)$ & \\
\hline ISS (median) & 17 (IQR: 9-25) & 20 (IQR: 17-34) & 0.027 \\
\hline NISS (median) & 17 (IQR: 12-29) & 29 (IQR: 17-36) & 0.022 \\
\hline \multicolumn{4}{|l|}{ Associated organ injury } \\
\hline Brain & $9(11.3 \%)$ & $4(23.5 \%)$ & 0.237 \\
\hline Chest & $26(32.5 \%)$ & $9(52.9 \%)$ & 0.111 \\
\hline Abdomen & $16(20.0 \%)$ & $6(35.3 \%)$ & 0.205 \\
\hline Urogenital & $12(15.0 \%)$ & $6(35.3 \%)$ & 0.080 \\
\hline Extremities & $47(58.8 \%)$ & $10(58.8 \%)$ & 0.996 \\
\hline OTA classification & & & 0.035 \\
\hline Stable ring & $8(10.0 \%)$ & 0 & \\
\hline Partially stable ring & $38(47.5 \%)$ & $5(29.4 \%)$ & \\
\hline Unstable ring & $34(42.5 \%)$ & $12(70.6 \%)$ & \\
\hline Osteosynthesis & & & 0.348 \\
\hline ORIF & $57(71.3 \%)$ & $14(82.4 \%)$ & \\
\hline CRIF or ESF & $23(28.7 \%)$ & $3(17.6 \%)$ & \\
\hline \multicolumn{4}{|l|}{ Radiological outcome } \\
\hline Matta/Tornetta criteria & & & 0.001 \\
\hline Excellent & $38(47.5 \%)$ & $4(23.5 \%)$ & \\
\hline Good & $27(33.8 \%)$ & $4(23.5 \%)$ & \\
\hline Fair & $15(18.8 \%)$ & $4(23.5 \%)$ & \\
\hline Poor & 0 & $5(29.4 \%)$ & \\
\hline Lefaivre criteria & & & 0.002 \\
\hline Excellent & $26(32.5 \%)$ & 0 & \\
\hline Good & $19(23.8 \%)$ & $2(11.8 \%)$ & \\
\hline Fair & $24(30.0 \%)$ & $7(41.2 \%)$ & \\
\hline Poor & $11(13.8 \%)$ & $8(47.1 \%)$ & \\
\hline Length of stay (days) & 17.3 (IQR: 12.5) & 32.3 (IQR: 12) & 0.001 \\
\hline Colostomy & $6(7.4 \%)$ & $5(29.4 \%)$ & 0.021 \\
\hline
\end{tabular}

IQR interquartile range, ISS injury severity score, NISS new injury severity score, ORIF open reduction and internal fixation, CRIF closed reduction and internal fixation, ESF external skeletal fixation

(17.5\%) patients were not. Thus, whether the pelvic fracture was open or closed remained uncorrelated with unsatisfactory outcomes at this time of evaluation. Further, multiple logistic regression analysis revealed that the potential factors related to dissatisfaction were fair-to-poor reduction quality using Matta/Tornetta and Lefaivre criteria (OR 3.79, 95\% CI 1.10-13.10 and OR 9.23, 95\% CI $1.71-49.75$, respectively) and experience with the diverting colostomy procedure (OR 7.69, 95\% CI 1.56-41.67) (Table 6).

\section{Discussion}

In this study, we found that patients with open pelvic fractures had comparable functional outcomes with those with closed fractures at the 1-year and 3-year evaluations. We identified poor radiological outcome (using Lefaivre criteria) and length of stay as potential factors for unsatisfactory 1-year functional outcomes. Regarding the 3-year functional evaluation, fair-to-poor radiological outcome (using Matta/Tornetta and Lefaivre criteria) and the 
Table 6 Results of multiple logistic regression analysis for risk factors associated with short-term and mid-term unsatisfactory functional outcomes

\begin{tabular}{|c|c|c|c|}
\hline \multirow[t]{2}{*}{ Risk factors } & \multicolumn{3}{|c|}{ Short-term functional outcome } \\
\hline & OR & Estimated $95 \% \mathrm{CI}$ & $p$ value \\
\hline Length of stay & 1.09 & $1.04-1.16$ & 0.001 \\
\hline \multicolumn{4}{|c|}{ Poor radiological outcome using } \\
\hline Lefaivre criteria & 3.55 & $1.07-11.76$ & 0.038 \\
\hline \multirow[t]{2}{*}{ Risk factors } & \multicolumn{3}{|c|}{ Mid-term functional outcome } \\
\hline & OR & Estimated $95 \% \mathrm{CI}$ & $p$ value \\
\hline Diverting colostomy & 7.69 & $1.56-41.67$ & 0.013 \\
\hline \multicolumn{4}{|c|}{ Fair-to-poor radiological outcome by } \\
\hline Matta/Tornetta criteria & 3.79 & $1.10-13.10$ & 0.035 \\
\hline Lefaivre criteria & 9.23 & $1.71-49.75$ & 0.010 \\
\hline
\end{tabular}

OR odds ratio, $C I$ confidence interval

presence of diverting colostomy were potential factors related to unsatisfactory functional outcome.

A higher injury severity score was a risk factor for poorer functional outcomes in previous studies $[8,9,15,30]$. Brouwers et al. reported that a high injury severity score was a prognostic factor for decreased healthrelated quality of life [15]. Patients with open pelvic fractures may hypothetically have a higher rate of mortalities and complications than those with closed fractures, as commonly reported in previous studies [6, 7, 31]. Frane et al. reported a mortality rate of $14 \%$, with an inclusive complication rate of $48.5 \%$ in patients with open pelvic fractures [32]. However, data regarding the functional status of survivors of open pelvic fractures are sparse. Although patients with an open fracture have more severe injuries than those with a closed fracture, we did not observe any difference in functional performance between these patients at the 1-year and 3-year evaluations. We postulated that the similar functional performance in both groups could be attributed to the application of adequate resuscitation, universal perioperative management, and patient-specific rehabilitation protocols.

Whether the quality of reduction for pelvic fracture is a key factor that may influence functional outcomes remains controversial [12, 33-36]. Kokubo et al. reported that a pelvic ring displacement of over $20 \mathrm{~mm}$ negatively influenced functional outcomes [10]. Nepola et al. stated that the degree of residual vertical displacement does not affect functional outcome [35]. The study results are consistent with those of the study by Kokubo et al. The statistical analysis revealed that the radiological outcomes of patients with both open and closed pelvic fractures had a significant effect on functional outcomes at the 1-year and 3-year follow-up. A worse postoperative function was correlated with a greater residual fracture gap and a bilaterally asymmetrical pelvis.

Another factor that might have affected functional performance in our study was the diverting colostomy procedure. Diverting colostomy is usually required in patients with concomitant colorectal injuries, especially those with open pelvic fractures [31, 37]. Studies have recommended that early diverting colostomy for open pelvic fractures, especially for Faringer zone I injury, is a crucial step in preventing surgical site infection, sepsis, and multiple organ dysfunction [6, 19, 37, 38]. Since diverting colostomy is a necessary procedure in patients with open pelvic fractures, there was no definite time for the colostomy closure. In our patients, diverting colostomy was usually performed while the patients could freely ambulate, usually 4-6 months after osteosynthesis. Although no significant relationship was observed between diverting colostomy and functional performance at the 1-year evaluation, diverting colostomy had a negative effect on functional outcome at the 1-year and 3-year evaluations. A possible reason was that patients who required diverting colostomy originally had more complex injuries than those who did not require this procedure. Therefore, the negative effect of pelvic fracture complexity was reflected by the diverting colostomy procedure.

Although we attempted to avoid bias, some limitations existed in our study. First, the retrospective design was inherently associated with the risk of recall bias, and the sample size was limited, because the data were obtained from a single institution. Second, some cases were exclu- 
ded, given the lack of complete functional evaluations for more than 36 months. Third, this study had a short followup duration. Fourth, although we attempted to apply universally acceptable perioperative management for these patients, the resuscitative procedures might not have been completely similar among the patients, including the necessity of trans-arterial embolization, timing of diversional colostomy, and analysis of osteosynthesis. Finally, different procedures may produce different results. Nevertheless, further studies with larger sample size and a prospective design are required.

In conclusion, open pelvic fracture was not an indicator of worse functional outcomes. Contrarily, patients with open pelvic fractures might have comparable functional outcomes with those with closed fractures at different time points within three years. Length of stay and the diverting colostomy procedure had negative effects on functional performance in the 1-year and 3-year evaluations, respectively. Furthermore, poor fracture reduction was correlated with worse functional outcomes at the two evaluation time points.

\section{Acknowledgements None.}

Author contributions C-YL was responsible for writing-original draft, data curation, formal analysis P-JL collected resources and was involved in validation. C-YS was involved in methodology. Y-HH was involved in visualization. Y-HY was involved in writing-review \& editing, conceptualization and project administration. C-YS, Y-CC and $\mathrm{Y}-\mathrm{HH}$ were involved in supervision. Y-CC and P-JL were involved in the investigation.

Funding This study did not receive any financial support or funding.

\section{Declarations}

Conflict of interest Each author certifies that he or she has no commercial associations that might pose a conflict of interest in connection with the submitted article.

Ethical approval The review process was approved by the Institutional Review Board (IRB No. 202101095B0).

Informed consent Acquisition of informed consent from patients was waived owing to the retrospective nature of this study.

Open Access This article is licensed under a Creative Commons Attribution 4.0 International License, which permits use, sharing, adaptation, distribution and reproduction in any medium or format, as long as you give appropriate credit to the original author(s) and the source, provide a link to the Creative Commons licence, and indicate if changes were made. The images or other third party material in this article are included in the article's Creative Commons licence, unless indicated otherwise in a credit line to the material. If material is not included in the article's Creative Commons licence and your intended use is not permitted by statutory regulation or exceeds the permitted use, you will need to obtain permission directly from the copyright holder. To view a copy of this licence, visit http://creativecommons. org/licenses/by/4.0/.

\section{References}

1. Korovessis P, Baikousis A, Stamatakis M, Katonis P (2000) Medium- and long-term results of open reduction and internal fixation for unstable pelvic ring fractures. Orthopedics 23:1165-1171. https://doi.org/10.3928/0147-7447-20001101-15

2. Martínez F, Alegret N, Carol F, Laso MJ, Zancajo J, García E, Ros V (2018) Pelvic fracture in the patient with multiple injuries: factors and lesions associated with mortality. Emergencias 30:91-97

3. Mi M, Kanakaris NK, Wu X, Giannoudis PV (2020) Management and outcomes of open pelvic fractures: an update. Injury S0020-1383:30170-30174. https://doi.org/10.1016/j.injury.2020. 02.096

4. Lai CY, Tseng IC, Su CY, Hsu YH, Chou YC, Chen HW, Yu YH (2020) High incidence of surgical site infection may be related to suboptimal case selection for non-selective arterial embolization during resuscitation of patients with pelvic fractures: a retrospective study. BMC Musculoskelet Disord 21:335. https://doi. org/10.1186/s12891-020-03372-5

5. Ghosh S, Aggarwal S, Kumar P, Kumar V (2019) Functional outcomes in pelvic fractures and the factors affecting them- A short term, prospective observational study at a tertiary care hospital. J Clin Orthop Trauma 10:896-899. https://doi.org/10. 1016/j.jcot.2018.08.001

6. Tseng IC, Chen IJ, Chou YC, Hsu YH, Yu YH (2020) Predictors of acute mortality after open pelvic fracture: experience from 37 patients from a level I trauma center. World J Surg 44:3737-3742. https://doi.org/10.1007/s00268-020-05675-z

7. Siada SS, Davis JW, Kaups KL, Dirks RC, Grannis KA (2017) Current outcomes of blunt open pelvic fractures: how modern advances in trauma care may decrease mortality. Trauma Surg Acute Care Open 2:e00136. https://doi.org/10.1136/tsaco-2017000136

8. Gabbe BJ, Hofstee DJ, Esser M, Bucknill A, Russ MK, Cameron PA, Handley C, de Steiger RN (2015) Functional and return to work outcomes following major trauma involving severe pelvic ring fracture. ANZ J Surg 85:749-754. https://doi.org/10.1111/ ans. 12700

9. Kabak S, Halici M, Tuncel M, Avsarogullari L, Baktir A, Basturk M (2003) Functional outcome of open reduction and internal fixation for completely unstable pelvic ring fractures (type C): a report of 40 cases. J Orthop Trauma 17:555-562. https://doi.org/ 10.1097/00005131-200309000-00003

10. Kokubo Y, Oki H, Sugita D, Takeno K, Miyazaki T, Negoro K, Nakajima H (2017) Functional outcome of patients with unstable pelvic ring fracture. J Orthop Surg (Hong Kong) 25:2309499016684322. https://doi.org/10.1177/ 2309499016684322

11. Sharpe JP, Magnotti LJ, Gobbell WC, Huang X, Perez EA, Fabian TC, Croce MA (2017) Impact of early operative pelvic fixation on long-term self-reported outcome following severe pelvic fracture. J Trauma Acute Care Surg 82:444-450. https:// doi.org/10.1097/TA.0000000000001346

12. Suzuki T, Shindo M, Soma K, Minehara H, Nakamura K, Uchino M, Itoman M (2007) Long-term functional outcome after unstable pelvic ring fracture. J Trauma 63:884-888. https://doi.org/10. 1097/01.ta.0000235888.90489.fc

13. Lefaivre KA, Slobogean GP, Valeriote J, O'Brien PJ, Macadam SA (2012) Reporting and interpretation of the functional outcomes after the surgical treatment of disruptions of the pelvic ring: a systematic review. J Bone Joint Surg Br 94:549-555. https://doi.org/10.1302/0301-620X.94B4.27960

14. Dienstknecht T, Pfeifer R, Horst K, Sellei RM, Berner A, Zelle BA, Probst C, Pape HC (2013) The long-term clinical outcome 
after pelvic ring injuries. Bone Joint J 95-B:548-553. https://doi. org/10.1302/0301-620X.95B4.30804

15. Brouwers L, de Jongh MAC, de Munter L, Edwards M, Lansink KWW (2020) Prognostic factors and quality of life after pelvic fractures. The brabant injury outcome surveillance (BIOS) study. PLoS ONE 15:e0233690. https://doi.org/10.1371/journal.pone. 0233690

16. Dente CJ, Feliciano DV, Rozycki GS, Wyrzykowski AD, Nicholas JM, Salomone JP, Ingram WL (2005) The outcome of open pelvic fractures in the modern era. Am J Surg 190:830-835. https://doi.org/10.1016/j.amjsurg.2005.05.050

17. Westhoff J, Höll S, Kälicke T, Muhr G, Kutscha-Lissberg F (2004) Open pelvic fracture: treatment strategy and results for 12 patients. Unfallchirurg 107:189-196. https://doi.org/10.1007/ s00113-003-0724-4

18. Meinberg EG, Agel J, Roberts CS, Karam MD, Kellam JF (2018) Fracture and dislocation classification compendium-2018. J Orthop Trauma 32(Suppl 1):S1-S170. https://doi.org/10.1097/ BOT.0000000000001063

19. Faringer PD, Mullins RJ, Feliciano PD, Duwelius PJ, Trunkey DD (1994) Selective fecal diversion in complex open pelvic fractures from blunt trauma. Arch Surg 129:958-963. https://doi. org/10.1001/archsurg.1994.01420330072014 963-964)

(Discussion

20. Jones AL, Powell JN, Kellam JF, McCormack RG, Dust W, Wimmer P (1997) Open pelvic fractures: a multicenter retrospective analysis. Orthop Clin North Am 28:345-350. https://doi. org/10.1016/s0030-5898(05)70293-5

21. Mataliotakis GI, Giannoudis PV (2011) Radiological measurements for postoperative evaluation of quality of reduction of unstable pelvic ring fractures: advantages and limitations. Injury 42:1395-1401. https://doi.org/10.1016/j.injury.2011.10.012

22. Lefaivre KA, Blachut PA, Starr AJ, Slobogean GP, O'Brien PJ (2014) Radiographic displacement in pelvic ring disruption: reliability of 3 previously described measurement techniques. J Orthop Trauma 28:160-166. https://doi.org/10.1097/BOT. 0b013e31829efcc5

23. Yu YH, Liu CH, Hsu YH, Chou YC, Chen IJ, Wu CC (2021) Matta's criteria may be useful for evaluating and predicting the reduction quality of simultaneous acetabular and ipsilateral pelvic ring fractures. BMC Musculoskelet Disord 22:544. https://doi. org/10.1186/s12891-021-04441-z

24. Tornetta P 3rd, Matta JM (1996) Outcome of operatively treated unstable posterior pelvic ring disruptions. Clin Orthop Relat Res 329:186-193. https://doi.org/10.1097/00003086-19960800000022

25. Pastor T, Tiziani S, Kasper CD, Pape HC, Osterhoff G (2019) Quality of reduction correlates with clinical outcome in pelvic ring fractures. Injury 50:1223-1226. https://doi.org/10.1016/j. injury.2019.04.015

26. Lefaivre KA, Starr AJ, Barker BP, Overturf S, Reinert CM (2009) Early experience with reduction of displaced disruption of the pelvic ring using a pelvic reduction frame. J Bone Joint Surg Br 91:1201-1207. https://doi.org/10.1302/0301-620X.91B9.22093
27. D'Aubigne RM, Postel M (1954) Functional results of hip arthroplasty with acrylic prosthesis. J Bone Joint Surg Am 36-A:451-475. https://doi.org/10.2106/00004623-19543603000001

28. d'Aubigné RM, Postel M (2009) The classic: functional results of hip arthroplasty with acrylic prosthesis. 1954. Clin Orthop Relat Res 467:7-27. https://doi.org/10.1007/s11999-008-0572-1

29. Majeed SA (1989) Grading the outcome of pelvic fractures. J Bone Joint Surg Br 71:304-306. https://doi.org/10.1302/0301620X.71B2.2925751

30. Harvey-Kelly KF, Kanakaris NK, Obakponovwe O, West RM, Giannoudis PV (2014) Quality of life and sexual function after traumatic pelvic fracture. J Orthop Trauma 28:28-35. https://doi. org/10.1097/BOT.0b013e31828fc063

31. Fu CY, Huang RY, Wang SY, Liao CH, Huang JF, Hsu YP, Lin CY, Kang SC (2018) Concomitant external and internal hemorrhage: challenges to managing patients with open pelvic fracture. Am J Emerg Med 36:1937-1942. https://doi.org/10.1016/j.ajem. 2018.02.014

32. Frane N, Iturriaga C, Bub C, Regala P, Katsigiorgis G, Linn M (2020) Risk factors for complications and in-hospital mortality: an analysis of 19,834 open pelvic ring fractures. J Clin Orthop Trauma 11:1110-1116. https://doi.org/10.1016/j.jcot.2020.08.017

33. Lindahl J, Hirvensalo E (2005) Outcome of operatively treated type-C injuries of the pelvic ring. Acta Orthop 76:667-678. https://doi.org/10.1080/17453670510041754

34. Pohlemann T, Bosch U, Gänsslen A, Tscherne H (1994) The Hannover experience in management of pelvic fractures. Clin Orthop Relat Res 305:69-80. https://doi.org/10.1097/00003086199408000-00010

35. Nepola JV, Trenhaile SW, Miranda MA, Butterfield SL, Fredericks DC, Riemer BL (1999) Vertical shear injuries: is there a relationship between residual displacement and functional outcome? J Trauma 46:1024-1029. https://doi.org/10.1097/ 00005373-199906000-00007 (Discussion 1029-1030)

36. Dujardin FH, Hossenbaccus M, Duparc F, Biga N, Thomine JM (1998) Long-term functional prognosis of posterior injuries in high-energy pelvic disruption. J Orthop Trauma 12:145-150. https://doi.org/10.1097/00005131-199803000-00001 (Discussion 150-151)

37. Fitzgerald CA, Moore TJ, Morse BC, Subramanian A, Dente CJ, Patel DC, Reisman WM, Schenker ML, Gelbard RB (2017) The role of diverting colostomy in traumatic blunt open pelvic fractures. Am Surg 83:e280-e282

38. Lunsjo K, Abu-Zidan FM (2006) Does colostomy prevent infection in open blunt pelvic fractures? A systematic review. J Trauma 60:1145-1148. https://doi.org/10.1097/01.ta. $0000197615.31553 .1 \mathrm{e}$

Publisher's Note Springer Nature remains neutral with regard to jurisdictional claims in published maps and institutional affiliations. 